\title{
The search for common pathways underlying asthma and COPD
}

This article was published in the following Dove Press journal:

International Journal of COPD

24 January 2013

Number of times this article has been viewed

\section{Yoshiko Kaneko \\ Yohei Yatagai \\ Hideyasu Yamada \\ Hiroki lijima \\ Hironori Masuko \\ Tohru Sakamoto \\ Nobuyuki Hizawa}

Department of Pulmonary Medicine, Faculty of Medicine, University of

Tsukuba, Tsukuba, Japan
Correspondence: Nobuyuki Hizawa Department of Pulmonary Medicine, University of Tsukuba, Tsukuba, Japan Tel/Fax +8I 298533 I44

EmalLnhizawa@md.tsukuba.ac.jp
Abstract: Recently, several genes and genetic loci associated with both asthma and chronic obstructive pulmonary disease (COPD) have been described as common susceptibility factors for the two diseases. In complex diseases such as asthma and COPD, a large number of molecular and cellular components may interact through complex networks involving gene-gene and gene-environment interactions. We sought to understand the functional and regulatory pathways that play central roles in the pathobiology of asthma and COPD and to understand the overlap between these pathways. We searched the PubMed database up to September 2012 to identify genes found to be associated with asthma, COPD, tuberculosis, or essential hypertension in at least two independent reports of candidate-gene associations or in genome-wide studies. To learn how the identified genes interact with each other and other cellular proteins, we conducted pathway-based analysis using Ingenuity Pathway Analysis software. We identified 108 genes and 58 genes that were significantly associated with asthma and COPD in at least two independent studies, respectively. These susceptibility genes were grouped into networks based on functional annotation: 12 (for asthma) and eleven (for COPD) networks were identified. Analysis of the networks for overlap between the two diseases revealed that the networks form a single complex network with 229 overlapping molecules. These overlapping molecules are significantly involved in canonical pathways including the "aryl hydrocarbon receptor signaling," "role of cytokines in mediating communication between immune cells," "glucocorticoid receptor signaling," and "IL-12 signaling and production in macrophages" pathways. The Jaccard similarity index for the comparison between asthma and COPD was 0.81 for the network-level comparison, and the odds ratio was $3.62(P<0.0001)$ for the asthma/COPD pair in comparison with the tuberculosis/ essential hypertension pair. In conclusion, although the identification of asthma and COPD networks is still far from complete, these networks may be used as frameworks for integrating other genome-scale information including expression profiling and phenotypic analysis. Network overlap between asthma and COPD may indicate significant overlap between the pathobiology of these two diseases, which are thought to be genetically related.

Keywords: COPD, asthma, network, common pathways, aryl hydrocarbon receptor signaling

\section{Introduction}

Both asthma and chronic obstructive pulmonary disease (COPD) are characterized by chronic inflammation and remodeling of the airways. ${ }^{1,2}$ A common pathogenetic basis for asthma and COPD is implied based on overlapping clinical characteristics, epidemiologic studies, and the association of genes common to both asthma and COPD. Genetics provides a unique tool for studying the pathophysiology of asthma and COPD. Traditional candidate gene studies may focus on a single gene or on a few genes in combination, with these genes identified on the basis of prior knowledge or on sus- 
pected pathogenetic mechanisms. In contrast, genome-wide association studies (GWAS) and linkage studies allow for the comprehensive evaluation of the entire genome without prior assumptions regarding the pathobiology. Nonetheless, the many genetic variations discovered can explain only a small fraction of the genetic risks associated with such complex diseases ${ }^{3}{ }^{3}$ complex biological systems and cellular networks may underlie most genotype-phenotype relationships. The high polygenicity of asthma and COPD, therefore, could suggest that genetic variants confer risk by functioning together within the same network, and that the functional unit conferring a disease risk may not be a single gene but rather the network itself. ${ }^{4}$

The Dutch hypothesis maintains that asthma and airway hyperresponsiveness predispose patients to developing COPD later in life and that asthma and COPD are different expressions of a single disease, ${ }^{5}$ which is based on the timing of environmental and epigenetic influences with a common genetic background. Host factors such as airway hyperresponsiveness, family history of asthma, and low lung function are common risk factors for asthma and COPD, as are environmental stimuli such as environmental tobacco smoke and air pollution. ${ }^{6,7}$ In recent decades, substantial progress has been made in characterizing the susceptible genes involved in asthma or COPD. Genes that have been implicated in both asthma and COPD include ADRB2, GSTM1, GSTP1, IL13, $T G F B 1, T N F, A D A M 33, C C L 5$, and $I L 17 F{ }^{8-13}$ Several common genetic predispositions, therefore, may contribute to the development of asthma and COPD, including predisposition to abnormal lung growth, resulting in lower lung function and delayed immune maturation; predisposition to lower respiratory viral infections and early allergic sensitization; and predisposition to bronchial hyperresponsiveness. By interacting with each other, genes and their products form complex cellular networks. Therefore, the Dutch hypothesis implies a polygenetic variation affecting the same pathway and the alteration of a functional network as a common root for increased risk for asthma and COPD.

The purpose of the current study was to identify the biological pathways and processes critical to asthma and COPD, and to analyze the genetic similarities between the two diseases using genes associated with asthma or COPD, as well as constructing interaction networks among those genes and their products. We used Ingenuity Pathway Analysis (IPA) software (Ingenuity Systems, Redwood City, CA, USA) to evaluate whether loci across the genome associated with asthma or COPD were enriched for connectivity among genes representing particular pathways or molecular processes. Assessing relatedness between diseases involves exploring the mechanisms that influence susceptibility and phenotype expression. Thus, the overlap of genes and their products could provide comparative insight into the pathogenetic mechanisms of asthma and COPD. The results from the current study will serve as a first step towards a better understanding of these chronic inflammatory lung diseases and the associated phenotypes with similar symptoms or modes of treatment.

\section{Materials and methods Database searches}

We systematically searched the results of gene association studies on asthma or COPD phenotypes that were published up to September 2012. We also searched disease-associated genes for two other diseases, tuberculosis (TB) and essential hypertension (E-HTN), as disease controls. We chose TB as a disease control because, like COPD and asthma, it too is characterized by chronic pulmonary inflammation. In contrast, we chose E-HTN as a negative disease control; it is highly unlikely that E-HTN shares any common pathogenesis with asthma, COPD, or TB. Both TB and E-HTN are also common complex diseases in which multiple genetic and environmental factors are involved in the etiology. In addition, for both diseases, genetic factors have been extensively studied, including in GWAS.

We selected genes associated with each disease that were reported in two or more independent association studies or demonstrated by GWAS. We identified the genes by searching the PubMed database using the keywords "association" and "SNP or polymorphism" with each of the following terms: "asthma," "COPD or chronic bronchitis or emphysema," "tuberculosis," and "essential hypertension." Search results were checked manually for relevance. The search was restricted to English-language and human studies. We also excluded pharmacogenetic studies from the search.

\section{Ingenuity pathway analysis}

We sought to identify asthma- and COPD-associated gene networks from these candidate genes. We used IPA software, which links specific genes to a database of gene functions gleaned from the biomedical research literature. IPA core analysis allows us to find interactions between genes and proteins, related networks, functions, and canonical pathways in the context of biological processes. Briefly, a set of genes identified by the PubMed search was uploaded into the web-delivered application and each gene identifier was 
mapped to its corresponding gene object in the Ingenuity Knowledge Base (Ingenuity Systems). Genes associated with a canonical pathway in the Ingenuity Knowledge Base were considered for the analysis. The significance of the association between the gene dataset and the canonical pathway was measured in two ways: (1) a ratio of the number of genes from the dataset that map to the pathway divided by the total number of molecules that exist in the canonical pathway; and (2) the Benjamini-Hochberg procedure for multiple testing correction, which allows us to calculate the false discovery rate for each of the probability values to determine whether the association between the genes in the dataset and the canonical pathway is explained by chance alone.

\section{IPA network generation}

Molecules of interest that interact with other molecules in the Ingenuity Knowledge Base are designated as network eligible molecules. IPA considers all network-eligible molecules on our gene list to be of equal importance when generating networks for molecule lists. Network-eligible molecules are combined into networks that maximize their specific connectivity, which is their interconnectedness with each other relative to all of the molecules with which they are connected in the Ingenuity Knowledge Base. Additional molecules from the Ingenuity Knowledge Base are used to specifically connect two or more smaller networks by merging them into a larger network. Networks are limited to 35 molecules each to keep them to a usable size. Networks are scored on the basis of the number of network-eligible molecules they contain. The score takes into account the number of networkeligible molecules in the network and its size, as well as the total number of network-eligible molecules analyzed, and the total number of molecules in the Ingenuity Knowledge Base that could potentially be included in the networks. In fact, the higher the score, the lower the probability of finding the observed number of network-eligible molecules in a given network by random chance. Focus molecules simply indicate the number of network-eligible molecules per network. The three most significant functions for each network are listed.

Canonical pathways are distinct from networks in that they are generated prior to data input, are based on the literature, and do not change upon data input, whereas networks are generated de novo on the basis of the researcher's own input data. Biological understanding of the function of genes in pathways, and the currently available lists of "canonical" pathways are evolving rapidly. In this study, five gene datasets were used to identify the most significantly associated canonical pathways: 108 genes for asthma, 58 genes for COPD, 37 genes for TB, 55 genes for E-HTN, and 229 genes common to both asthma and COPD.

Table I Genes associated with BA, COPD, TB, or E-HTN

BA ACAAI, ACE, ADAM33, ADRB2, ALOX5AP, ARGI, ARG2, ATG5, ATPAFI, BDNF, C3, C5, CAT, CCLII, CCL24, CCL5, CCR2, CCR3, CDI4, CFTR, CHI3LI, CHIA, CMAI, COX2, CRTAM, CTLA4, CYSLTRI, CYSLTR2, DEFBI, DENNDIB, DPPI0, EDNI, FCER2, FLG, GSDML, GSTMI, GSTPI, GSTTI, HAVCRI, HAVCR2, HHIP, HLA-DPAI, HLA-DQBI, HLA-DRBI, IFNG, ILIO, ILI 2B, ILI 3, ILI I, ILI 7, ILI 7F, ILI 8, ILI 8RI, ILIB, ILIRA, ILIRLI, IL2RB, IL33, IL4, IL4R, IL6R, IL8, IRFI, ITGB3, LRRC32, LTA, LTA4H, LTC4S, MBL, MS4A2, MUC7, MYLK, NAT2, NOSI, NOS3, NPSRI, NPSRI, NQOI, ORMDL3, PAII, PERLDI, PHFII, PLA2G7, PPARG, PTGDR, PTGDR2, PTGER2, PTGER3, RAD50, RORA, SCGBIAI, SMAD3, PINK5, STAT3, STAT6, TBX2I, TBXA2R, TGFBI, TLR2, TLR4, TLR6, TLR9, TNC, TNF, TNFA, TSLP, VDR, VEGF

COPD ABCCI, ACE, ADAMI9, ADAM33, ADRB2, AGER, BICDI, CHRNA3, CHRNA5, CNTN5, CSF2, CTLA4, CYP2A6, DBP, EDNI, EPHXI, ESRI, FAMI IA, FGF7, GC, GSTCD, GSTMI, GSTO2, GSTPI, HHIP, HMOXI, HTR4, ILI3, ILIB, IL6, INTSI 2, IREB2, LEP, MACROD2, MMPI, MMPI2, MMP9, MSRI, NFKBIB, NOS3, NPNT, PDE4D, PPT2, SERPINAI, SERPINE2, SFTPB, SFTPD, SIRT2, SOD3, STATI, TGFBI, THSD4, TLR4, TNFA, TNSI, TP53, TRPV4, XRCCI

TB CCLI 7, CCL2, CCL5, CD209, CRI, CTSZ, DYNLRB2, EBFI, HAUS6, HLA-DQB, HLA-DRBI, IFNG, IFNGRI, ILI0, ILI 2B, ILI 2RBI, ILIB, IL4, JAGI, MBL, MC3R, MIF, NOS2A, NRAMPI, P2RX7, PENK, SLCIIAI, TAPI, TLR2, TLR8, TLR9, TMEFF2, TNFA, TNFRSFIB, TXNDC4, VDR, WTI

E-HTN ACE, ACE2, ADDI, ADD2, ADRB2, AGT, AGTRI, AGTRLI, ALDH2, APOE, ATPIBI, BDKRB2, CACNAIH, CALCA, CAT, CYPIIB2, CYP2J2, CYP4AII, EDN2, FRS2, GNB3, GRK4, GSTTI, HMOXI, HSDI IB2, HSD3BI, INSR, IPO7, KLKI, LYZ, M6PR, MTHFR, NEDD4L, NOS3, NR3C2, PMSI, PNMT, PRCI, PRKGI, REN, RGS2, RGS5, SELE, SLC24A3, SLC24A4, SLC4AI, SLC8AI, SV2B, TGFB, TH, TSC, WNKI, WNK4, WSCD2, YEATS4

Note: The official Entrez Gene name is used for each gene (http://www.ncbi.nlm.nih.gov/gquery).

Abbreviations: BA, bronchial asthma; COPD, chronic obstructive pulmonary disease; TB, tuberculosis; E-HTN, essential hypertension; ACE, angiotensin I converting enzyme; BDNF, brain-derived neutrophic factor; $C 3$, complement component 3; C5, complement component 5; CAT, catalase; CCL, chemokine (C-C) ligand; CCR, chemokine (C-C) receptor; CFTR, cystic fibrosis transmembrane conductance regulator; CHIA, chitinase; FLG, filaggrin; GST, glutathionine S-transferase; HLA, major histocompatibility complex; IFNG, interferon gamma; IL, interleukin; NOS, nitric oxide synthase; TNC, tenascin C; TNF, tumor necrosis factor; TSLP, thymic stromal lymphopoietin; VDR, vitamin D receptor; VEGF, vascular endothelial growth factor; FGF, fibroblast growth factor; HHIP, hedgehog interacting protein; MMP, matrix metalloproteinase; NFkB, nuclear factor kappa B; TGF, transforming growth factor; AGT, angiotensinogen; APOE, apolipoprotein E; ATP, adenosine triphosphate; REN, renin; SELE, selectin E; $\mathrm{TH}$, thyrosine hydroxylase. 


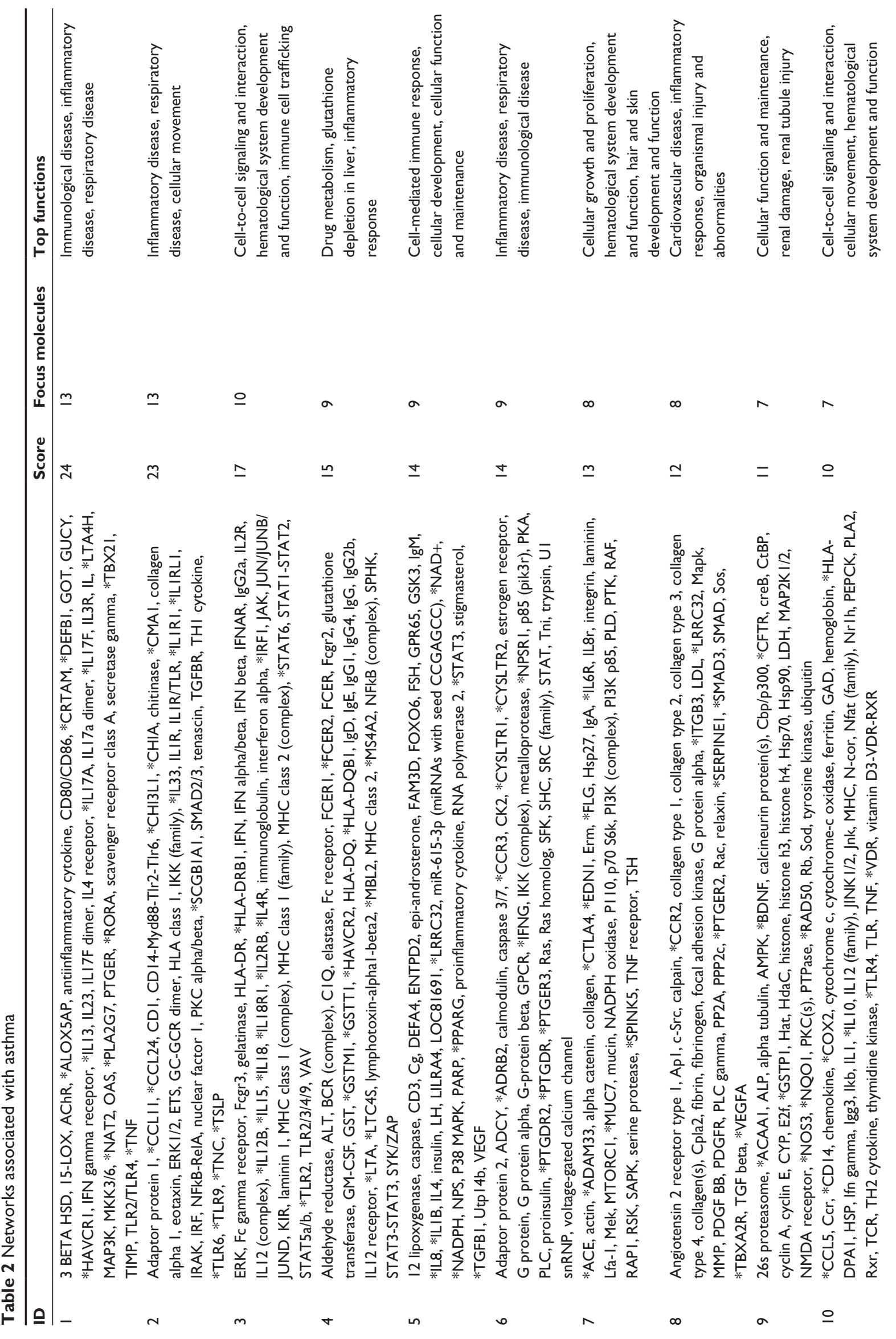




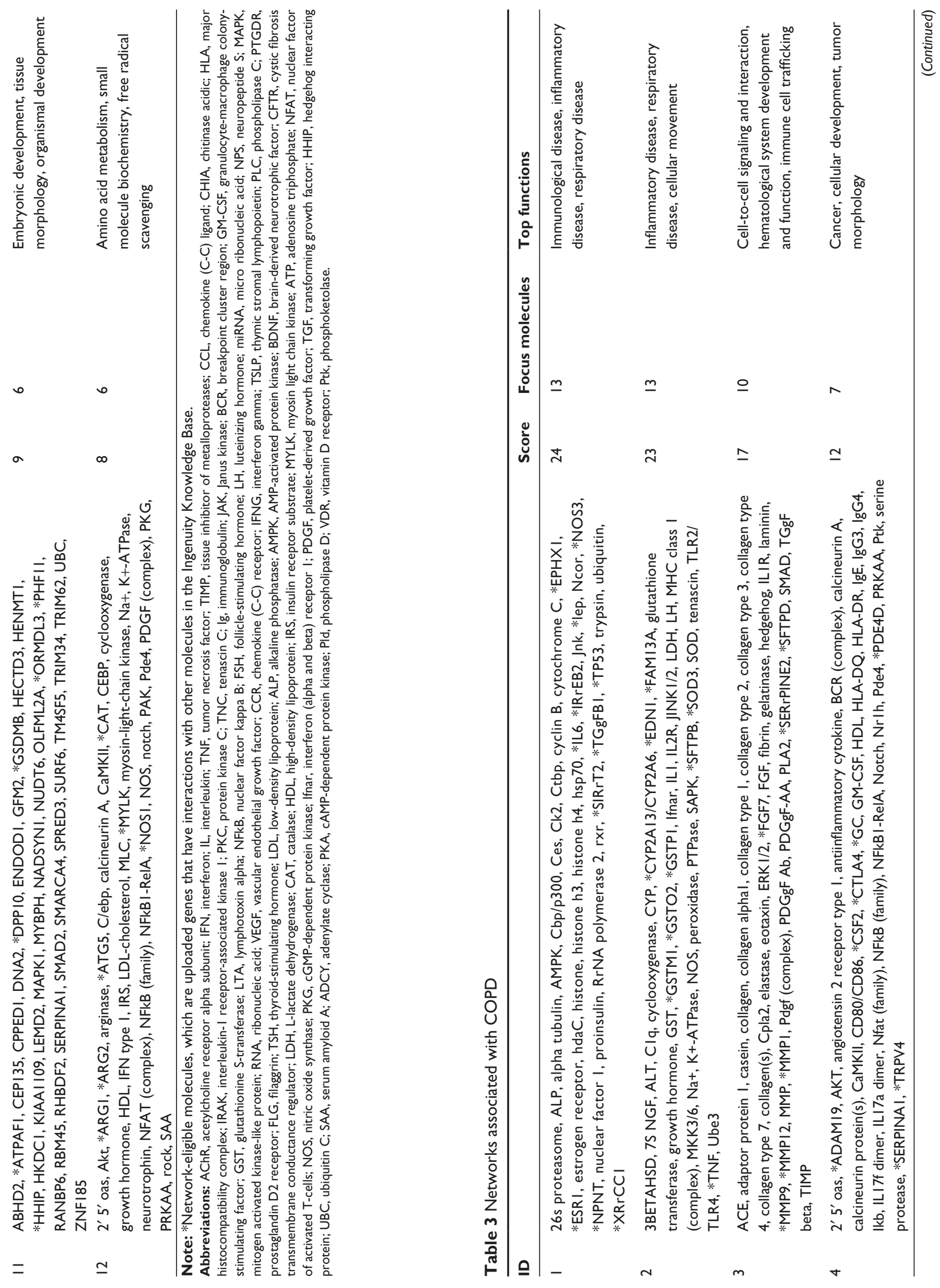




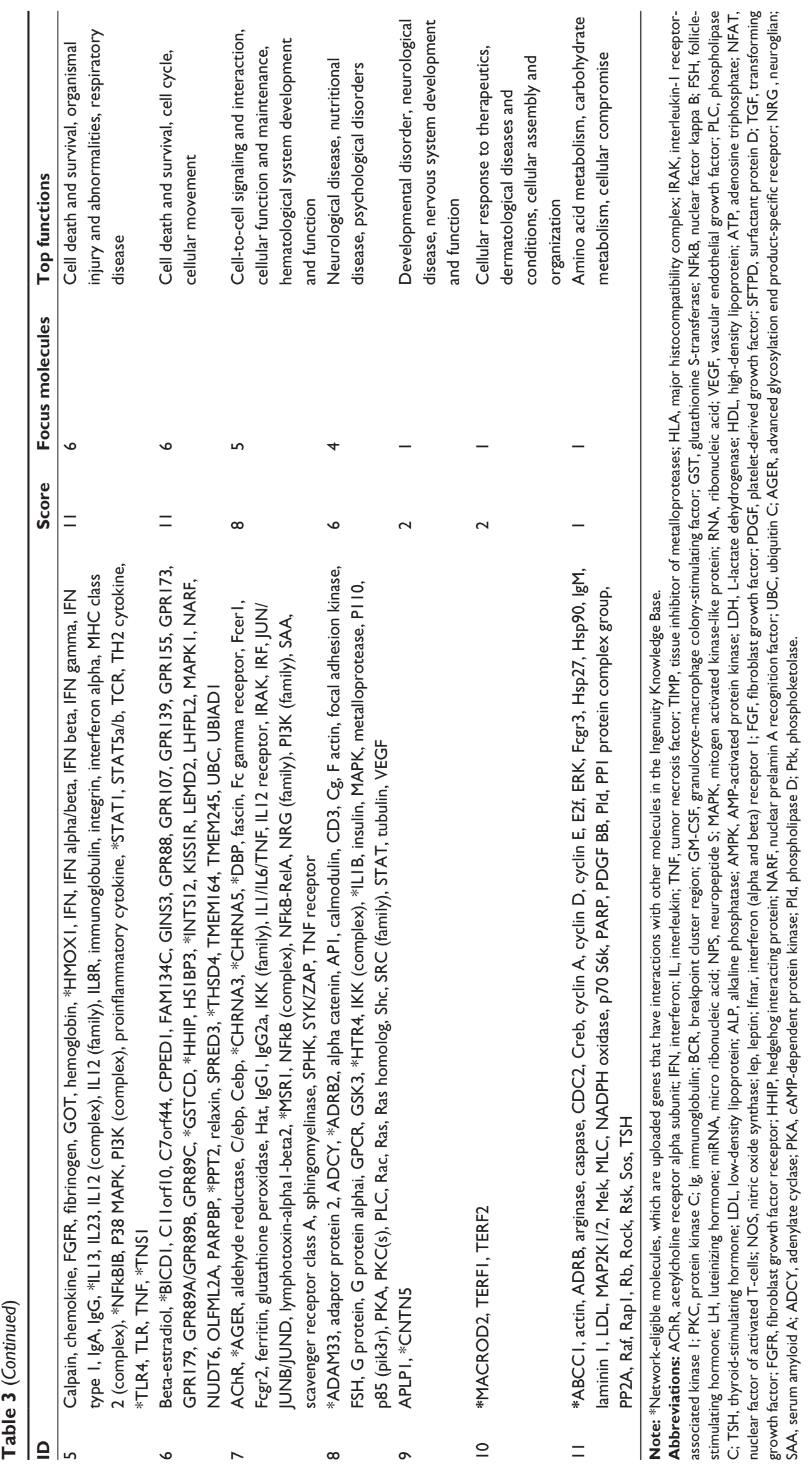




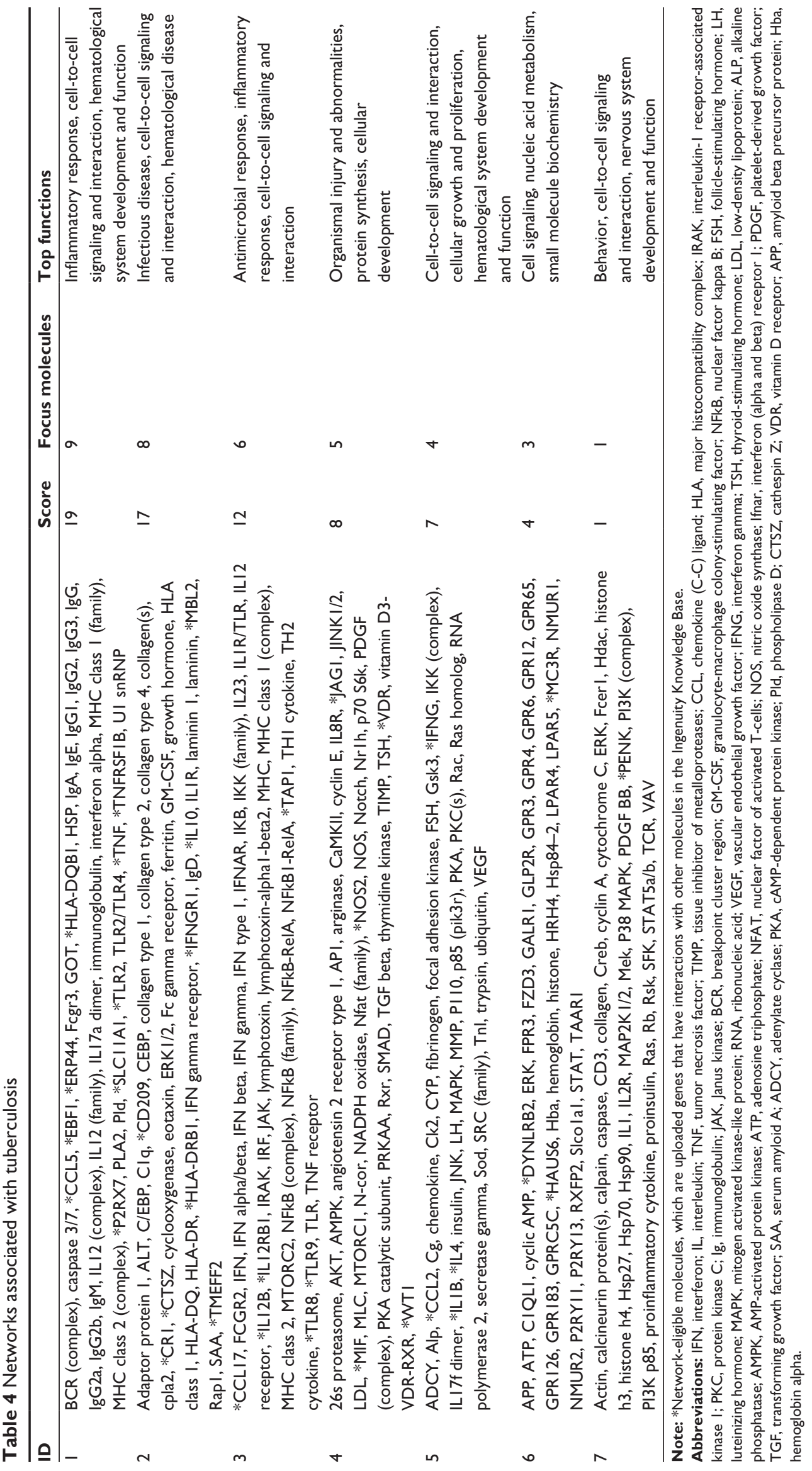




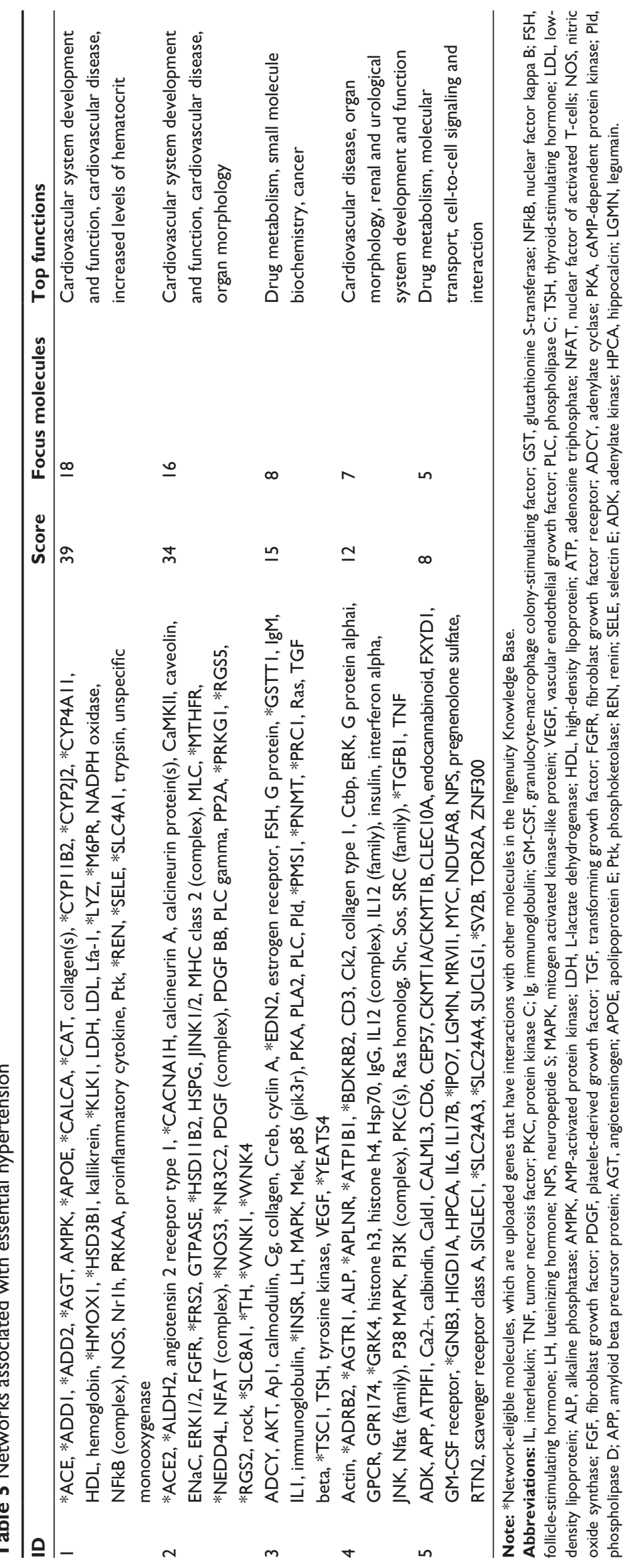




\section{Relatedness between asthma and COPD}

A pairwise comparison of asthma and COPD and of TB and E-HTN was performed at the network level. The Jaccard ${ }^{14}$ similarity index was used to measure the degree of association between the two diseases. This index considers the similarity between two diseases as the number of genes shared divided by the total number of genes present in either of them. It may be expressed as follows:

$$
\mathrm{J}=\mathrm{C} /(\mathrm{A}+\mathrm{B}-\mathrm{C})
$$

where $\mathrm{A}$ is the number of genes present in a given disease $\mathrm{A}$; $\mathrm{B}$ is the number of genes present in disease $\mathrm{B}$; and $\mathrm{C}$ is the number of genes present in both disease $\mathrm{A}$ and disease $\mathrm{B}$. The number of genes present in either of the diseases is given by $\mathrm{A}+\mathrm{B}-\mathrm{C}$. To assess the significance of the relatedness in network-level comparisons, we compared the Jaccard similarity index for asthma and COPD with that for TB and E-HTN as the reference because it is highly unlikely that TB and E-HTN are genetically related. Using a $2 \times 2$ contingency table for the two groups of disease comparisons, each was sorted according to the genes that were common or unique to the diseases; the odds ratios (ORs) and associated significance levels were calculated.

\section{Results}

The PubMed search identified 108 asthma-, 58 COPD-, 37 TB-, and 55 E-HTN-associated genes (Table 1). Using information on these genes, the IPA program created 12 , eleven, seven, and five networks for these diseases, respectively (Tables 2-5). The networks for each disease consisted of 419, 320, 244, and 175 genes or their products for asthma, COPD, TB, and E-HTN, respectively.

\section{Biological pathway analysis (IPA)}

For asthma, the main canonical pathways identified were the "T-helper cell differentiation," "altered T-cell and B-cell signaling in rheumatoid arthritis," "role of cytokines in mediating communication between immune cells," and "communication between innate and adaptive immune cells" pathways (Table 6). For COPD, the main pathways identified were the "hepatic fibrosis/hepatic stellate cell activation," "aryl hydrocarbon receptor signaling," "glucocorticoid receptor signaling," and "differential regulation of cytokine production in macrophages and T-helper cells by IL-17A and IL-17F" pathways (Table 6).

The canonical pathway "aryl hydrocarbon receptor signaling" was most significantly associated with the overlap of gene datasets between asthma and COPD, followed by multiple canonical pathways that showed highly significant associations such as the "role of cytokines in mediating communication between immune cells," "glucocorticoid receptor signaling," "IL-12 signaling and production in macrophages," and "hepatic fibrosis/hepatic stellate cell activation" pathways (Table 7).

Twelve asthma and eleven COPD networks generated a large single network (Figure 1); 229 genes were common to both diseases, and 190 and 91 genes were unique to asthma and COPD, respectively. The numbers of network genes common or unique to each disease comparison are shown in Table 8. The Jaccard similarity index for the network level comparison between asthma and COPD was 0.81 . The OR was $3.62(P<0.0001)$ for the asthma/COPD pair in comparison with the TB/E-HTN pair (Table 9). The Jaccard similarity index for the comparisons between asthma and TB and between COPD and TB were 0.67 (OR 2.98; $P<0.0001$ ) and 0.63 (OR 2.79; $P<0.0001$ ), respectively (Table 9).

\section{Discussion}

IPA is a software program that helps researchers model, analyze, and understand complex systems by integrating data from a variety of experimental platforms and providing insight into molecular and chemical interactions, cellular phenotypes, and disease processes. To expand upon the understanding of the genetic architecture and molecular basis of asthma and COPD, we used the IPA program to evaluate whether loci across the genomes previously associated with asthma or COPD were enriched for connectivity among genes representing particular pathways or molecular processes. We observed a significant overlap between asthma- and COPD-associated genetic loci, which may reflect the significant epidemiologic and clinical overlap between the two diseases. The common and distinct functional and regulatory pathways identified in this study may play central roles in the pathophysiology of asthma and COPD, which could help us to understand the primary pathogenesis underlying these diseases.

The literature provides good evidence that the canonical pathways highlighted in the current study could lead to the development of both asthma and COPD. The aryl hydrocarbon receptor (AhR) is a ligand-dependent transcription factor whose activity is modulated by xenobiotics as well as by physiologic ligands. The AhR is an attenuator of pulmonary inflammation caused by tobacco smoke, regulating pathogenic processes implicated in COPD etiology and progression, inflammation, and cell death. ${ }^{15}$ It also modulates allergic inflammatory responses. Mast cells, located at the 
Table 6 Top ten canonical pathways significantly associated with each group of disease susceptibility genes

\begin{tabular}{|c|c|c|c|c|}
\hline & & B-H P & Ratio & Molecules in the pathway \\
\hline \multicolumn{5}{|c|}{ BA pathways } \\
\hline 1 & T-helper cell differentiation & I.26E-24 & $2.5 \mathrm{E}-0 \mathrm{I}$ & $\begin{array}{l}\text { IFNG, STAT6, IL4R, ILI0, IL6R, HLA-DRBI, STAT3, HLA- } \\
\text { DQBI, ILI3, TBX2I, ILI8RI, ILI7A, ILI8, TGFBI, ILI2B, } \\
\text { ILI7F, TNF, IL4 }\end{array}$ \\
\hline 2 & $\begin{array}{l}\text { Altered T-cell and B-cell signaling in } \\
\text { rheumatoid arthritis }\end{array}$ & $7.94 \mathrm{E}-23$ & $1.96 \mathrm{E}-0 \mathrm{I}$ & $\begin{array}{l}\text { IFNG, ILI0, ILI5, HLA-DRBI, HLA-DQBI, TLR9, ILI7A, IL33, } \\
\text { TLR2, TLR4, ILI8, TGFBI, ILI I2B, LTA, TLR6, ILIB, TNF, IL4 }\end{array}$ \\
\hline 3 & $\begin{array}{l}\text { Role of cytokines in mediating communication } \\
\text { between immune cells }\end{array}$ & $2.00 \mathrm{E}-19$ & $2.55 \mathrm{E}-0 \mathrm{I}$ & $\begin{array}{l}\text { IL8, IFNG, ILI0, ILI5, ILI3, ILI7A, IL33, ILI8, ILI2B, TGFBI, } \\
\text { ILIB, ILI7F, TNF, IL4 }\end{array}$ \\
\hline 4 & $\begin{array}{l}\text { Communication between innate } \\
\text { and adaptive immune cells }\end{array}$ & $3.98 \mathrm{E}-19$ & I.47E-0 I & $\begin{array}{l}\text { IFNG, IL8, ILI0, ILI5, HLA-DRBI, CCL5, TLR9, IL33, TLR2, } \\
\text { TLR4, ILI8, ILI2B, TLR6, ILIB, TNF, IL4 }\end{array}$ \\
\hline 5 & Hepatic fibrosis/hepatic stellate cell activation & $2.5 \mathrm{IE}-17$ & I.I6E-0I & $\begin{array}{l}\text { IFNG, IL8, IL4R, ILIRLI, ILI0, SMAD3, IL6R, ILIRI, CCL5, } \\
\text { VEGFA, TLR4, EDNI, TGFBI, CDI4, ILIB, TNF, IL4 }\end{array}$ \\
\hline 6 & $\begin{array}{l}\text { Differential regulation of cytokine production in } \\
\text { intestinal epithelial cells by IL- I7A and IL-I7F }\end{array}$ & IE-I6 & 4.35E-0I & $\begin{array}{l}\text { IFNG, ILI0, ILI2B, ILIB, DEFBI, CCL5, ILI3, ILI7F, TNF, } \\
\text { ILI7A }\end{array}$ \\
\hline 7 & $\begin{array}{l}\text { Role of macrophages, fibroblasts, and } \\
\text { endothelial cells in rheumatoid arthritis }\end{array}$ & $3.98 \mathrm{E}-16$ & 6.3 IE-02 & $\begin{array}{l}\text { IL8, ILIRLI, ILI0, ILI5, IL6R, STAT3, ILIRI, CCL5, TLR9, } \\
\text { ILI8RI, ILI7A, TLR2, VEGFA, IL33, TLR4, ILI8, TGFBI, LTA, } \\
\text { TLR6, ILIB, TNF }\end{array}$ \\
\hline 8 & $\begin{array}{l}\text { Differential regulation of cytokine production } \\
\text { in macrophages and T-helper cells by } \\
\text { IL-I7A and IL-I7F }\end{array}$ & IE-I3 & 4.44E-0I & ILI0, ILI2B, ILIB, CCL5, ILI3, ILI7F, TNF, ILI7A \\
\hline 9 & $\begin{array}{l}\text { Role of hypercytokinemia/hyperchemokinemia } \\
\text { in the pathogenesis of influenza }\end{array}$ & IE-I3 & 2.27E-0I & IL33, IL8, IFNG, ILI8, ILI2B, ILI5, ILIB, CCL5, TNF, ILI7A \\
\hline 10 & IL-I0 signaling & IE-I2 & I.4IE-0I & $\begin{array}{l}\text { IL33, ILI8, IL4R, ILI0, ILIRLI, CDI4, ILIB, ARG2, STAT3, } \\
\text { ILIRI, TNF }\end{array}$ \\
\hline
\end{tabular}

\section{COPD pathways}

I Hepatic fibrosis/hepatic stellate cell activation

2.04E-08 6.85E-02 TLR4, LEP, EDNI, TGFBI, ILIB, IL6, STATI, TNF, MMP9, MMPI

$2 \quad$ Aryl hydrocarbon receptor signaling

3 Glucocorticoid receptor signaling

I.86E-07 5.59E-02

TP53, GSTMI, TGFBI, ILIB, IL6, GSTO2, TNF, ESRI, GSTPI

I.86E-07 3.74E-02 TGFBI, ILIB, IL6, STATI, CSF2, NFkBIB, ILI3, TNF, ESRI, MMPI, ADRB2

4 Differential regulation of cytokine production

$2.88 \mathrm{E}-07 \quad 2.78 \mathrm{E}-0$

ILIB, IL6, CSF2, ILI3, TNF

in macrophages and T-helper cells by

IL-I7A and IL-I7F

$5 \quad$ Atherosclerosis signaling

$6 \quad L X R / R X R$ activation

7 Role of cytokines in mediating communication between immune cells

8 Colorectal cancer metastasis signaling

$\begin{array}{ll}6.03 \mathrm{E}-06 & 3.5 \mathrm{E}-02 \\ & \\ 6.17 \mathrm{E}-06 & 3.86 \mathrm{E}-02 \\ 0.000016 & 6.52 \mathrm{E}-02\end{array}$

$\begin{array}{lll}5.0 I E-07 & 5.88 E-02 & \text { MSRI, TGFBI, ILIB, SERPINAI, IL6, TNF, MMP9, MMPI } \\ 5.75 E-07 & 5.88 E-02 & \text { TLR4, MSRI, ILIB, SERPINA I, IL6, GC, TNF, MMP9 }\end{array}$

I.38E-06 I.09E-0I TGFBI, ILIB, IL6, CSF2, ILI 3, TNF

\section{Dendritic cell maturation}

I0 Altered T-cell and B-cell signaling in rheumatoid arthritis

TP53, TLR4, TGFBI, IL6, STATI, MMPI2, TNF, MMP9, MMPI

TLR4, LEP, ILIB, IL6, STATI, CSF2, NFkBIB, TNF

TLR4, TGFBI, ILIB, IL6, CSF2, TNF

\section{TB pathways} rheumatoid arthritis

2 Communication between innate and 5.0IE-I5 I.0IE-0I adaptive immune cells

3 T-helper cell differentiation

I.26E-I4 I.39E-0I

TLR2, IFNG, ILI0, ILI2B, TLR8, HLA-DRBI, ILIB, HLADQBI, TNF, TLR9, IL4

TLR2, IFNG, ILI0, ILI2B, TLR8, HLA-DRBI, ILIB, CCL5, TNF, TLR9, IL4

IFNG, ILIO, ILI 2B, ILI2RBI, HLA-DRBI, IFNGRI, HLADQBI, TNFRSFIB, TNF, IL4

Note: B-H P; the IPA computes FDRs from P-values using the Benjamini-Hochberg procedure.

Abbreviations: BA, bronchial asthma; IFNG, interferon gamma; IL, interleukin; HLA, major histocompatibility complex;TGF, transforming growth factor;TLR,Toll-like receptor; LTA, lymphotoxin alpha;TNF, tumor necrosis factor; CCL, chemokine (C-C) ligand;VEGF, vascular endothelial growth factor; EDN, endothelin; ARG, arginase; COPD, chronic obstructive pulmonary disease; LEP, leptin; MMP, matrix metalloproteinase; GST, glutathionine S-transferase; ESR, esterase 5 regulator; CSF2, colony-stimulating factor 2; MSR, methionine sulfoxide reductase; LXR, LexA regulated function; RXR, retinoid X receptor; NFkBIB, nuclear factor of kappa light polypeptide gene enhancer in $B$ cells inhibitor beta; TB, tuberculosis; NOS, nitric oxide synthase; E-HTN, essential hypertension; TH, tyrosine hydroxylase; PNMT, phenylethanolamine N-methyltransferase; REN, renin; ACE, angiotensin I converting enzyme; AGT, angiotensinogen; APOE, apolipoprotein E; LYZ, lysozyme; SELE, selectin E; cAMP, cyclic adenosine monophosphate; GRK, glycerate kinase;APLNR, apelin receptor;AGTRI, angiotension 2 receptor type I;ADRB2, adrenoceptor beta 2; INSR, insulin receptor;ALDH2, aldehyde dehydrogenase 2; CAT, catalase; GNB3, guanine nucleotide binding protein beta 3; IPA, Ingenuity Pathway Analysis; FDRs, false discovery rates. 
Table 6 (Continued)

\begin{tabular}{|c|c|c|c|c|}
\hline & & B-H P & Ratio & Molecules in the pathway \\
\hline 4 & $\begin{array}{l}\text { Differential regulation of cytokine production in } \\
\text { intestinal epithelial cells by IL-I7A and IL- I7F }\end{array}$ & $1.58 \mathrm{E}-12$ & 3.04E-0I & IFNG, CCL2, ILI0, ILI2B, ILIB, CCL5, TNF \\
\hline 5 & $\begin{array}{l}\text { Role of pattern recognition receptors in } \\
\text { recognition of bacteria and viruses }\end{array}$ & I.58E-I I & 8.49E-02 & TLR2, MBL2, ILI0, ILI2B, TLR8, ILIB, CCL5, TNF, TLR9 \\
\hline 6 & $\begin{array}{l}\text { Differential regulation of cytokine production } \\
\text { in macrophages and T-helper cells by } \\
\text { IL-I7A and IL-I7F }\end{array}$ & $3.98 \mathrm{E}-\mathrm{II}$ & 3.33E-0I & CCL2, ILI0, ILI2B, ILIB, CCL5, TNF \\
\hline 7 & Type I diabetes mellitus signaling & $3.98 \mathrm{E}-1 \mathrm{I}$ & 7.44E-02 & $\begin{array}{l}\text { IFNG, ILI2B, HLA-DRBI, ILIB, IFNGRI, HLA-DQBI, } \\
\text { TNFRSFIB, NOS2, TNF }\end{array}$ \\
\hline 8 & IL-I 2 signaling and production in macrophages & $2.29 \mathrm{E}-10$ & 5.77E-02 & TLR2, IFNG, ILI0, IL I 2B, ILI 2RB I, IFNGR I, NOS2, TNF, IL4 \\
\hline 9 & $\begin{array}{l}\text { Crosstalk between dendritic cells and } \\
\text { natural killer cells }\end{array}$ & $2.5 \mathrm{IE}-10$ & $8.42 \mathrm{E}-02$ & CD209, IFNG, ILI 2B, HLA-DRBI, TNFRSFI B, TNF, TLR9, IL4 \\
\hline 10 & Hepatic fibrosis/hepatic stellate cell activation & $2.88 \mathrm{E}-10$ & $6.16 \mathrm{E}-02$ & IFNG, CCL2, ILI0, ILIB, IFNGRI, CCL5, TNFRSFIB, TNF, IL4 \\
\hline \multicolumn{5}{|c|}{ E-HTN pathways } \\
\hline I & Catecholamine biosynthesis & 0.013 & I.33E-0I & TH, PNMT \\
\hline 2 & Mineralocorticoid biosynthesis & 0.027 & $9.52 \mathrm{E}-02$ & CYPIIB2, HSD3BI \\
\hline 3 & Glucocorticoid biosynthesis & 0.027 & $9.52 \mathrm{E}-02$ & CYPIIB2, HSD3BI \\
\hline 4 & Renin-angiotensin signaling & 0.027 & $3.2 \mathrm{E}-02$ & REN, AGTRI, ACE, AGT \\
\hline 5 & Atherosclerosis signaling & 0.027 & $2.94 \mathrm{E}-02$ & APOE, LYZ, SELE, TGFBI \\
\hline 6 & cAMP-mediated signaling & 0.027 & $2.26 \mathrm{E}-02$ & GRK4, RGS2, APLNR, AGTRI, ADRB2 \\
\hline 7 & AMPK signaling & 0.03 & 2.4IE-02 & TSCI, INSR, NOS3, ADRB2 \\
\hline 8 & Ethanol degradation 4 & 0.035 & $6.9 \mathrm{E}-02$ & ALDH2, CAT \\
\hline 9 & Protein kinase A signaling & 0.041 & I.47E-02 & TH, GNB3, ADD2, TGFBI, ADDI, NOS3 \\
\hline 10 & Glucocorticoid receptor signaling & 0.041 & I.7E-02 & SELE, TGFBI, NR3C2, AGT, ADRB2 \\
\hline
\end{tabular}

boundaries between tissues and the external environment, are a potential target of AhR ligands. Murine and human mast cells constitutively express $\mathrm{AhR}$, and its activation by the high-affinity ligand 6-formylindolo[3,2-b]carbazole induces a boost in degranulation. Moreover, AhR-activated mast cells produce IL-17, a critical player in chronic inflammation and autoimmunity, suggesting a novel pathway for mast cell activation in the pathogenesis of asthma and COPD. ${ }^{16}$

The "differential regulation of cytokine production in macrophages and T-helper cells and epithelial cells by

Table 7 Canonical pathways significantly associated with genes common to both BA and COPD

\begin{tabular}{|c|c|c|c|c|}
\hline \multicolumn{2}{|c|}{ BA/COPD common pathways } & \multirow{2}{*}{$\begin{array}{l}\text { B-H P } \\
2.70 \mathrm{E}-06\end{array}$} & \multirow{2}{*}{$\begin{array}{l}\text { Ratio } \\
6.83 \mathrm{E}-02\end{array}$} & \multirow{2}{*}{$\begin{array}{l}\text { Molecules in the pathway } \\
\text { Rxr, GSTMI, ApI, MAPKI, TGFBI, NFkB, ILIB, TNF, } \\
\text { GSTPI, Hsp90, NFkB-RelA }\end{array}$} \\
\hline I & Aryl hydrocarbon receptor signaling & & & \\
\hline 2 & $\begin{array}{l}\text { Role of cytokines in mediating communication } \\
\text { between immune cells }\end{array}$ & 0.000022 & 7.27E-02 & TGFBI, ILIB, ILI3, TNF \\
\hline 3 & Glucocorticoid receptor signaling & 0.000022 & $5.44 \mathrm{E}-02$ & $\begin{array}{l}\text { MAPKI, PRKAA, Hsp70, histone h3, ILI3, lkb, Hsp90, } \\
\text { PII0, RNA polymerase 2, TGFBI, NFkBI-RelA, NFkB, } \\
\text { STAT5a/b, ILIB, TNF, ADRB2 }\end{array}$ \\
\hline 4 & IL-I2 signaling and production in macrophages & 0.000022 & $6.4 I E-02$ & $\begin{array}{l}\text { TLR4, PI I0, NFkB, MAPKI, TGFBI, SERPINAI, Ikb, } \\
\text { TNF, NFkB-RelA }\end{array}$ \\
\hline 5 & Hepatic fibrosis/hepatic stellate cell activation & 0.000022 & 4.79E-02 & TLR4, EDNI, TGFBI, NFkB, ILIB, TNF, NFkB-RelA \\
\hline 6 & TREMI signaling & 0.000026 & $8.45 \mathrm{E}-02$ & TLR4, MAPKI, NFkB, ILIB, TNF, NFkB-RelA \\
\hline 7 & $\begin{array}{l}\text { Differential regulation of cytokine production in } \\
\text { macrophages and T-helper cells by IL-I7A and IL-I7F }\end{array}$ & 0.000038 & I.67E-0I & ILIB, ILI3, TNF \\
\hline 8 & $\begin{array}{l}\text { Differential regulation of cytokine production in } \\
\text { intestinal epithelial cells by IL-I7A and IL-I7F }\end{array}$ & 0.000069 & $1.3 \mathrm{E}-0 \mathrm{I}$ & ILIB, ILI3, TNF \\
\hline 9 & Altered T-cell and B-cell signaling in rheumatoid arthritis & 0.000069 & 8.7E-02 & TLR4, CD3, TGFBI, NFkB, ILIB, TNF, NFkB-RelA, IgM \\
\hline 10 & $\begin{array}{l}\text { Role of pattern recognition receptors in recognition of } \\
\text { bacteria and viruses }\end{array}$ & 0.000093 & $6.6 \mathrm{E}-02$ & TLR4, PIIO, MAPKI, NFkB, ILIB, TNF, NFkB-RelA \\
\hline
\end{tabular}

Note: B-H P; the IPA computes FDRs from P-values using the Benjamini-Hochberg procedure.

Abbreviations: BA, bronchial asthma; COPD, chronic obstructive pulmonary disease; Rxr, retinoid X receptor; GST, glutathionine S-transferase; MAPK, mitogen activated kinase-like protein; TGFB, transforming growth factor beta; NFkB, nuclear factor kappa B; IL, interleukin; TNF, tumor necrosis factor; RNA, ribonucleic acid; ADRB2, adrenoceptor beta 2; TLR, Toll-like receptor; TGF, transforming growth factor; Ig, immunoglobulin; IPA, Ingenuity Pathway Analysis; FDRs, false discovery rates. 


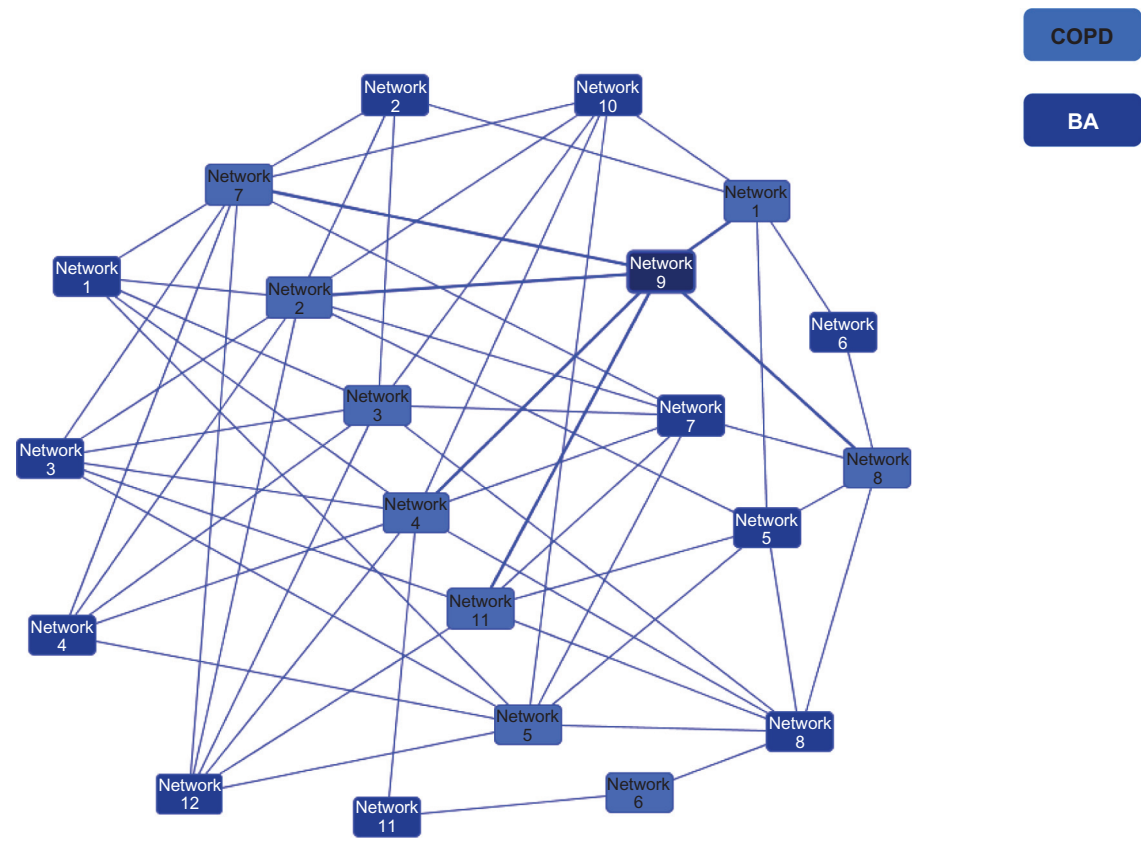

Figure I Overlapping networks between asthma and COPD.

Notes: The IPA program identified 229 overlapping molecules between 12 BA networks and eleven COPD networks and then merged them into a single larger network. Each network is represented by a colored rectangle and is labeled with its corresponding network number. Two COPD networks (numbers 9 and I0) had no overlapping molecules with any of the BA networks and are not depicted.

Abbreviations: COPD, chronic obstructive pulmonary disease; BA, bronchial asthma; IPA, Ingenuity Pathway Analysis.

IL-17A and IL-17F" pathway (see Table 7) has also been identified as a gene dataset common to both asthma and COPD. Neutrophilic airway inflammation is a common feature of COPD and is recognized in asthma, particularly in severe disease. The Th17 cytokines IL-17A and IL-17F have been implicated in the development of neutrophilic airway inflammation, and increased expressions of these cytokines have been implicated in asthma and COPD. ${ }^{17}$ Notably, tobacco smoke is a selective adjuvant that augments in vitro and in vivo Th17 cell differentiation via the AhR, suggesting that tobacco smoke is a potent Th17 adjuvant, and that IL-17RA signaling is required for the chemokine expression necessary for MMP12 induction and tissue emphysema. ${ }^{18}$

In addition, identification of the canonical pathways for a common gene dataset such as the "role of cytokines in mediating communication between immune cells," "role of pattern recognition receptors in recognition of bacteria and viruses," and "IL-12 signaling and production in macrophages" pathways (see Table 7) also suggest that asthma and COPD share common genetic associations related to impaired innate and adaptive immunity. These key pathways may be disrupted via many different causes - genetic, epigenetic, and environmental - in patients with asthma or COPD. Even if the disease arises from a different specific cause in different individuals, the disease in each of these individuals could nonetheless share disruption of these related key biological processes.

In the obtained disease networks, asthma and COPD belong to a single interconnected main giant component (Figure 1), which is consistent with the idea that asthma and COPD are much more connected to each other than hitherto believed. Therefore, asthma and COPD could be viewed as perturbations of highly interlinked cellular networks. For chronic inflammatory lung disease, polygenetic variation alters the behaviors of a biological pathway in response to environmental exposures including allergens, infections (bacterial or viral), tobacco smoke, and ambient or indoor air pollution. Functional alterations of any single node through function-modifying mutation either may have no effect at all, owing to the emergent network property of robustness, or, for network hubs, may yield a phenotype such as asthma or COPD. The heterogeneity between asthma and COPD could be due, at least in part, to mutations in different genes having similar phenotypic effects owing to their acting on the same functional pathway. The functional pathways identified for the genes common to asthma and COPD, therefore, could play a role in the proposed association between asthma and COPD.

Although patients with asthma or COPD are believed to have no increased risk for TB, TB showed more similarities 
Table 8 Numbers of genes and related molecules that are unique or common to given disease pairs

\begin{tabular}{lllll}
\hline Disease A & $\begin{array}{l}\text { Molecules } \\
\text { unique to } \\
\text { disease A }\end{array}$ & $\begin{array}{l}\text { Molecules } \\
\text { common to } \\
\text { both diseases }\end{array}$ & $\begin{array}{l}\text { Molecules } \\
\text { unique to } \\
\text { disease B }\end{array}$ & Disease B \\
\hline TB & 179 & 65 & 110 & E-HTN \\
BA & 190 & 229 & 91 & COPD \\
TB & 54 & 190 & 229 & BA \\
TB & 87 & 157 & 163 & COPD \\
E-HTN & 84 & 91 & 328 & BA \\
E-HTN & 88 & 87 & 233 & COPD \\
\hline
\end{tabular}

Abbreviations: $\mathrm{TB}$, tuberculosis; $\mathrm{BA}$, bronchial asthma; $\mathrm{COPD}$, chronic obstructive pulmonary disease; E-HTN, essential hypertension.

to asthma and COPD than did E-HTN. This may reflect the fact that these three inflammatory disorders are characterized by chronic inflammation in the lung, sharing common immune and inflammatory networks for their pathogenesis. In this study, many canonical pathways related to innate and adaptive immune responses were commonly identified for the networks identified in asthma, COPD, and TB, suggesting the important role of nonspecific inflammatory responses in asthma and COPD as well as in TB, which are mediated by cytokines and chemokines induced by the interaction of innate receptors expressed in macrophages and dendritic cells.

We acknowledge that there are inherent biases in such literature reviews, given the reduced likelihood of negative studies being published. Moreover, if the first published study yields a negative result, other investigators are less likely to study that gene. However, because even the most replicated genes have one or more negative studies, a gene with an initial negative result could still be a true susceptibility gene in other populations, or with respect to phenotypes or variants other than those studied in the initial report. In addition, we did not take into account the size of the study samples, the effect size, the overall quality of the analysis, or whether

Table 9 The Jaccard similarity index calculated for each disease pair

\begin{tabular}{lllll}
\hline & BA & COPD & TB & E-HTN \\
\hline BA & - & & & \\
COPD & $0.81(3.62$, & - & & \\
& $<0.000 I)$ & & & \\
TB & $0.67(2.98$, & $0.63(2.79$, & - & \\
& $<0.000 I)$ & $<0.000 I)$ & & - \\
E-HTN & $0.21(0.98,0.92)$ & $0.27(1.2,0.31)$ & 0.22 \\
& & & (reference) & \\
\hline
\end{tabular}

Notes: The Jaccard similarity index (OR, $P$-value) is shown for each disease comparison. $O R s$ and their associated $P$-values were calculated for a given disease comparison and were compared with the TB/E-HTN comparison.

Abbreviations: BA, bronchial asthma; COPD, chronic obstructive pulmonary disease; TB, tuberculosis; E-HTN, essential hypertension; OR, odds ratio. the genotype data were in Hardy-Weinberg proportions. Rather, we reported positive findings when the investigators interpreted their results as supporting an association, and we reported negative findings when the investigators interpreted their results as not supporting an association.

\section{Conclusion}

The current study suggests that the genetic contribution to chronic inflammatory lung diseases operates through multiple genes interacting in different functional pathways, providing insight into similarities in the underlying pathogenetic mechanisms between asthma and COPD. Environmental factors including allergens, infections, and smoking may alter the expression and regulation of common networks that mediate risk for asthma and COPD. Identification of networks shared by asthma and COPD may prove useful in the diagnosis and treatment of these two diseases. Although the link between these two diseases warrants further study, we anticipate that these genetic insights will transform the landscape of common complex diseases such as asthma and COPD.

\section{Acknowledgments}

We thank Mrs Flaminia Miyamasu, an associate professor at the Faculty of Medicine, University of Tsukuba, Tsukuba, Japan, who proofread and commented on this paper. The study was partly supported by a Grant-in-Aid for Scientific Research (B), No 24390206, from the Japan Society for the Promotion of Science.

\section{Disclosure}

The authors report no conflicts of interest in this work.

\section{References}

1. Barnes PJ. Immunology of asthma and chronic obstructive pulmonary disease. Nat Rev Immunol. 2008;8(3):183-192.

2. Gelb AF, Zamel N, Krishnan A. Physiologic similarities and differences between asthma and chronic obstructive pulmonary disease. Curr Opin Pulm Med. 2008;14(1):24-30.

3. Vercelli D. Discovering susceptibility genes for asthma and allergy. Nat Rev Immunol. 2008;8(3):169-182.

4. Sullivan PF. Puzzling over schizophrenia: schizophrenia as a pathway disease. Nat Med. 2012;18(2):210-211.

5. Orie NG. The Dutch hypothesis. Chest. 2000;117(5 Suppl 1):299S.

6. Brutsche MH, Downs SH, Schindler C, et al; SAPALDIA Team. Bronchial hyperresponsiveness and the development of asthma and COPD in asymptomatic individuals: SAPALDIA cohort study. Thorax. 2006;61(8):671-677.

7. Svanes C, Sunyer J, Plana E, et al. Early life origins of chronic obstructive pulmonary disease. Thorax. 2010;65(1):14-20.

8. Hizawa N, Yamaguchi E, Konno S, Tanino Y, Jinushi E, Nishimura M. A functional polymorphism in the RANTES gene promoter is associated with the development of late-onset asthma. Am J Respir Crit Care Med 2002;166(5):686-690. 
9. Hizawa N, Kawaguchi M, Huang SK, Nishimura M. Role of interleukin17F in chronic inflammatory and allergic lung disease. Clin Expl Allergy. 2006;36(9):1109-1114.

10. Van Eerdewegh P, Little RD, Dupuis J, et al. Association of the ADAM33 gene with asthma and bronchial hyperresponsiveness. Nature. 2002;418(6896):426-430.

11. van Diemen CC, Postma DS, Vonk JM, Bruinenberg M, Schouten JP, Boezen HM. A disintegrin and metalloprotease 33 polymorphisms and lung function decline in the general population. Am J Respir Crit Care Med. 2005;172(3):329-333.

12. Hizawa N, Makita H, Nasuhara Y, et al; Hokkaido COPD Cohort Study Group. Functional single nucleotide polymorphisms of the CCL5 gene and nonemphysematous phenotype in COPD patients. Eur Respir J. 2008;32(2):372-378.

13. Postma DS, Kerkhof M, Boezen HM, Koppelman GH. Asthma and chronic obstructive pulmonary disease: common genes, common environments? Am J Respir Crit Care Med. 2011;183(12):1588-1594.
14. Jaccard P. The distribution of the flora in the alpine zone. New Phytol. 1912;11(2):37-50.

15. Rico de Souza A, Zago M, Pollock SJ, Sime PJ, Phipps RP, Baglole CJ. Genetic ablation of the aryl hydrocarbon receptor causes cigarette smoke-induced mitochondrial dysfunction and apoptosis. J Biol Chem. 2011;286(50):43214-43228.

16. Sibilano R, Frossi B, Calvaruso M, et al. The aryl hydrocarbon receptor modulates acute and late mast cell responses. J Immunol. 2012; 189(1):120-127.

17. Doe C, Bafadhel M, Siddiqui S, et al. Expression of the T helper 17-associated cytokines IL-17A and IL-17F in asthma and COPD. Chest. 2010;138(5):1140-1147.

18. Chen K, Pociask DA, McAleer JP, et al. IL-17RA is required for CCL2 expression, macrophage recruitment, and emphysema in response to cigarette smoke. PLoS One. 2011;6(5):e20333.

\section{Publish your work in this journal}

The International Journal of COPD is an international, peer-reviewed journal of therapeutics and pharmacology focusing on concise rapid reporting of clinical studies and reviews in COPD. Special focus is given to the pathophysiological processes underlying the disease, intervention programs, patient focused education, and self management protocols.

\section{Dovepress}

This journal is indexed on PubMed Central, MedLine and CAS. The manuscript management system is completely online and includes a very quick and fair peer-review system, which is all easy to use. Visit http://www.dovepress.com/testimonials.php to read real quotes from published authors. 